\title{
Salmonella Typhimurium infection disrupts but continuous feeding of Bacillus based probiotic restores gut microbiota in infected hens
}

\author{
Samiullah Khan and Kapil K. Chousalkar *iD
}

\begin{abstract}
Background: The gut microbiota plays an important role in the colonisation resistance and invasion of pathogens. Salmonella Typhimurium has the potential to establish a niche by displacing the microbiota in the chicken gut causing continuous faecal shedding that can result in contaminated eggs or egg products. In the current study, we investigated the dynamics of gut microbiota in laying chickens during Salmonella Typhimurium infection. The optimisation of the use of an infeed probiotic supplement for restoration of gut microbial balance and reduction of Salmonella Typhimurium load was also investigated.
\end{abstract}

Results: Salmonella infection caused dysbiosis by decreasing (FDR $<0.05)$ the abundance of microbial genera, such as Blautia, Enorma, Faecalibacterium, Shuttleworthia, Sellimonas, Intestinimonas and Subdoligranulum and increasing the abundance of genera such as Butyricicoccus, Erysipelatoclostridium, Oscillibacter and Flavonifractor. The higher Salmonella Typhimurium load resulted in lower $(P<0.05)$ abundance of genera such as Lactobacillus, Alistipes, Bifidobacterium, Butyricimonas, Faecalibacterium and Romboutsia suggesting Salmonella driven gut microbiota dysbiosis. Higher Salmonella load led to increased abundance of genera such as Caproiciproducens, Acetanaerobacterium, Akkermansia, Erysipelatoclostridium, Eisenbergiella, EscherichiaShigella and Flavonifractor suggesting a positive interaction of these genera with Salmonella in the displaced gut microbiota. Probiotic supplementation improved the gut microbiota by balancing the abundance of most of the genera displaced by the Salmonella challenge with clearer effects observed with continuous supplementation of the probiotic. The levels of acetate and butyrate in the faeces were not affected $(P>0.05)$ by Salmonella challenge and the butyrate level was increased by the continuous feeding of the probiotic. Probiotic supplementation in Salmonella challenged chickens resulted in higher level of propionate. Continuous probiotic supplementation decreased $(P<0.05)$ the overall mean load of Salmonella in faeces and had a significant effect on Salmonella load reduction in internal organs.

Conclusions: Salmonella challenge negatively impacts the diversity and abundance of many gut microbial genera involved in important functions such as organic acid and vitamin production. Strategic feeding of a Bacillus based probiotic helps in restoring many of the microbial genera displaced by Salmonella Typhimurium challenge.

Keywords: $16 \mathrm{~S}$ rRNA sequencing, Chicken gut microbiota, Gut metabolites, Salmonella Typhimurium, Strategic feeding of probiotic

\footnotetext{
* Correspondence: Kapil.chousalkar@adelaide.edu.au

School of Animal and Veterinary Sciences, The University of Adelaide

Roseworthy, South Australia 5371, Australia
}

(c) The Author(s). 2020 Open Access This article is distributed under the terms of the Creative Commons Attribution 4.0 International License (http://creativecommons.org/licenses/by/4.0/), which permits unrestricted use, distribution, and reproduction in any medium, provided you give appropriate credit to the original author(s) and the source, provide a link to the Creative Commons license, and indicate if changes were made. The Creative Commons Public Domain Dedication waiver (http://creativecommons.org/publicdomain/zero/1.0/) applies to the data made available in this article, unless otherwise stated. 


\section{Background}

The chicken gut microbiome is composed of multiple microorganisms and their genetic materials. These microorganisms (microbiota) are involved in functions that are critical to bird health and performance. The gut microbiota help in digestion and metabolism [1], regulation of enterocytes [2], vitamin synthesis and development and regulation of the host immune system [3]. The chicken gut microbiota is mainly composed of the phyla Proteobacteria, Bacteroidetes, Actinobacteria and Firmicutes [4]. The host gut microbiota is affected by multiple factors such as disease, diet, husbandry conditions and age [5]. Salmonella Typhimurium causes clinical disease in many animals and humans; however, chickens are often asymptomatic carriers. Pathogenic Salmonella present in laying production systems often result in gastroenteritis in humans after the consumption of contaminated food [6]. In the chicken gut, Salmonella elicits inflammation through the activation of Salmonella pathogenicity island 1 (SPI1) for encoding the type III secretion system [7]. In the inflamed gut, motility allows Salmonella Typhimurium to utilize available nutrients for its enhanced growth [8]. To escape nutrient limitation caused by the intestinal microbiota, Salmonella uses specific metabolic traits for the utilisation of compounds that are not metabolized by gut microbiota [9].

Gram-negative bacteria dominate the gut at an early age, while gram positive Firmicutes, particularly Clostridia taxa, become more prominent at later ages [10]. A previous study demonstrated a negative correlation between Enterobacteriaceae and Lachnospiraceae, Ruminococcaceae, Erysipelotrichaceae and Peptostreptococcaceae in Salmonella Enteritidis challenged chicks [11], thus causing gut dysbiosis. Gut dysbiosis results from microbial imbalance due to impaired microbiota [12]. The mechanism by which the gut microbiome affects pathogen colonisation is partly mediated by the production of short-chain fatty acids (SCFAs) that are the metabolites of bacterial fermentation of undigested dietary fibre [13]. SCFAs activate G protein-coupled receptors (GPCRs) including free fatty-acid receptors 2 and 3 (FFAR2 and FFAR3) [14], inhibit histone deacetylases [15] and provide energy to enterocytes [16]. Although the roles of GPCRs (e.g. FFAR3/GPR41 and FFAR2/GPR43) are not well established, they have been implicated in the regulation of leukocytes [17] and leptin production [18] in murine models. GPR41 and GPR43 play a role in lowering body weight through the down-regulation of leptin mRNA [18]. Previous research in broiler chickens showed that the activation of GPR41 and GPR43 by gut microbiota derived SCFAs resulted in the production of Glucagon-like peptide-1 (GLP-1), which supressed lipid accumulation in the liver [19]. Therefore, the host microbiome constitutes an attractive target for manipulation, as it can be modified for pathogen colonisation resistance to reduce disease risk.

To strengthen and improve the gut microbiota composition in chickens, pre- and pro- biotics are often supplemented as a part of the feeding regimen. Prebiotics are host non-digestible complex carbohydrates that help to increase the resident gut microbiota through fermentation. Examples of prebiotics are pectin, xylooligosaccharides, galactooligosaccharides, fructooligosaccharides and inulin. Probiotics are live microbial feed supplements that beneficially affect the host by improving its intestinal microbial balance [20]. The representative bacterial genera in probiotics include Lactobacillus, Bifidobacterium, Enterococcus, Streptococcus and Bacillus. Apart from gram-positive bacteria, some probiotics are also composed of yeast and moulds. Some of the proposed functions of probiotics include competitive exclusion [21], antagonism [22], bacterial interference [23], barrier effect [24], modulation of host immune system [25] and colonization resistance [26]. These actions are achieved mainly through bacteria-bacteria and hostbacteria interactions. The bacteria-bacteria interactions result in the production of SCFAs [27], modification of redox potential [28], production of antimicrobial compounds, competition for epithelial receptors, quorum sensing [29] and production of an ecosystem harmful for pathogenic organisms. The reduced luminal $\mathrm{pH}$ due to organic acids restricts the growth of many pathogens. Probiotic bacteria secrete enzymes that hydrolyse bacterial toxins and modify toxin receptors [30]. Attachment of probiotic bacteria to cell surface receptors of enterocytes initiates signalling events that result in the synthesis of cytokines [31] and stimulation of toll-like receptors [32].

In laying hens, probiotics are generally used as feed supplements for improving flock performance and egg quality $[33,34]$. From the food safety perspective, Salmonella is an important foodborne pathogen that is often present in the gut of chickens. Salmonella reduction in layers, for the production of safer egg and egg products, has always been a priority for the egg industry. In Australia, Salmonella Typhimurium has been responsible for the majority of the egg related foodborne outbreaks [6]. The supplemental use of probiotics lowers the incidence of Salmonella in poultry production [35]. Given the longer commercial life span of egg laying hens, in order to achieve the cost-effective reduction in Salmonella shedding, it is critical to optimise the use of probiotics and to understand the dynamics of gut microbiota during probiotic treatment. Previous studies of probiotics use for Salmonella control in laying chickens have mainly focused either on young chicks, using different serovars of Salmonella or have used a short duration trial where the effect of Salmonella was not tested on 
gut microbiota dysbiosis at different time-points while chickens were laying [36-38]. In this study, we raised Salmonella free birds to understand the role of Salmonella Typhimurium in gut microbiota dysbiosis and its subsequent restoration through the use of a Bacillus based probiotic in laying chickens from point of laying until 30 weeks of age. Based on the role of microbiota in the clearance of gut pathogens, we hypothesised that, if used strategically, a Bacillus based probiotic could be effective in positively modulating the microbiota for gut health during Salmonella Typhimurium infection.

\section{Methods}

\section{Ethics approval}

All experimental work was approved by the Animal Ethics Committee at The University of Adelaide under approval number S-2017-080 in accordance with the guidelines specified in "Australian code for the care and use of animals for scientific purposes, 8th edition (2013)".

\section{Rearing of laying chickens}

Eggs from an Isa-Brown parent breeder flock were obtained from a hatchery, fumigated and hatched at the School of Animal and Veterinary Sciences. Meconium samples were tested through standard culture methods for the presence of Salmonella spp. (if any). Before placement of day-old laying chicks, the rearing facility was tested for the presence of Salmonella spp. The dayold female chicks were divided into six treatment groups (7 chickens in each treatment group), reared in pens until week 14 and then transferred into individual cages. The treatment groups were: negative control (NC), Salmonella challenge (SX), continuous probiotic supplemented and Salmonella challenge (CPX), continuous probiotic supplemented control (CPC), intermittent probiotic supplemented and Salmonella challenge (IPX) and intermittent probiotic control (IPC). The feeding regime was as per the protocol of the ISA General Management Guide. Before adding the probiotic, the feed was fumigated and regularly tested for the presence of Salmonella. For the probiotic-supplemented groups, $1 \mathrm{~g}$ of Bacillus based probiotic (Bacillus subtilis DSM 32324, Bacillus subtilis DSM 32325 and Bacillus amyloliquefaciens) was mixed with $1 \mathrm{~kg}$ of fumigated feed. The intermittent probiotic supplemented groups were on the probiotic supplement for alternate 4 weeks (4 weeks ON/ OFF strategy). Faeces from all the treatment groups were tested fortnightly for Salmonella isolation until the specific group chickens were challenged with Salmonella Typhimurium. At 18 weeks of age, pullets from the selected groups were orally inoculated with $10^{6}$ colony forming units (CFUs) per mL of Salmonella Typhimurium phage type 9, while the control groups received phosphate buffered saline (PBS). For the preparation of bacterial inoculum, Salmonella Typhimurium was grown on xylose lysine deoxycholate (XLD; ThermoFisher Scientific, Australia) agar and a single colony was subcultured in Luria-Bertani (LB) broth. The inoculum was prepared by re-suspending the washed bacterial pellet in PBS. Ten-fold serial dilutions of the original inoculum were plated onto XLD to confirm the CFU received by the individual chickens.

\section{Faecal shedding profile of Salmonella Typhimurium challenged chickens}

Individual chickens were monitored for the faecal shedding profile of Salmonella Typhimurium by sampling the faeces on days 3, 5 and 7 and then weeks 2, 4, 6, 8, 10 and 12 post-challenge. Fresh faecal samples were collected in sterile zip lock bags from individual chickens including the control groups. Faecal samples were also collected in $1.5 \mathrm{~mL}$ and $5 \mathrm{~mL}$ tubes and stored at $-80^{\circ} \mathrm{C}$ until used for microbial DNA extraction and quantification of SCFAs, respectively. The SCFAs analysis was performed on samples collected at weeks 1, 4, 8 and 12 post-challenge. A miniaturized most probable number (mMPN) method was used for the enumeration of Salmonella Typhimurium in individual positive faecal samples. The mMPN method was originally developed by the USDA-FDA, validated on chicken faecal samples for Salmonella enumeration [39] and has been used frequently in similar studies [40, 41]. The bacterial culture and $\mathrm{mMPN}$ procedures were performed following the methods previously described [40].

\section{Processing of eggs for Salmonella enumeration}

Once the chickens were in lay, eggs from all the treatment groups were aseptically collected every fortnight in Whirl Pack plastic bags and processed for the enumeration of Salmonella Typhimurium on the eggshell surface and in egg internal contents following the methods previously described $[41,42]$. An mMPN was performed on the samples positive for Salmonella Typhimurium.

\section{Short chain fatty acids quantification in faeces}

The faecal samples stored at $-80{ }^{\circ} \mathrm{C}$ ( $\leq 3$ month-old samples) were processed for SCFAs (acetate, propionate and butyrate) quantification using gas chromatography (Hewlett-Packard6890; Palo Alto, CA, USA) equipped with a BP21 capillary column $10 \mathrm{~mm}$, I.D. $0.32 \mathrm{~mm}$, film thickness $0.25 \mathrm{~mm}$ (SGE Pty Ltd., Australia) and a flame ionisation detector (FID). Briefly, $0.1 \mathrm{~g}$ of individual faecal samples were weighed into $1.5 \mathrm{~mL}$ centrifuge tubes into which $1 \mathrm{~mL}$ of water containing $2 \%$ orthophosphoric acid was dispensed. A $20-\mu \mathrm{L}$ of internal standard ( $1 \mathrm{mmol} / \mathrm{L}$ of 4 -methyl valerate) was added to each sample which was then briefly vortexed and incubated for $30 \mathrm{~min}$ at room temperature. The samples were 
centrifuged at $12,000 \mathrm{r} / \mathrm{min}$ for $10 \mathrm{~min}$. The supernatants were transferred with a disposable glass Pasteur pipette to their corresponding $6 \mathrm{~mL}$ scintillation vials and $2 \mathrm{~mL}$ of diethyl ether was added into each sample which was then briefly vortexed. The upper layer of diethyl ether was transferred into corresponding gas chromatography vials and run for SCFAs analysis. A programmed temperature ramp $\left(50-220^{\circ} \mathrm{C}\right)$ was used. Helium gas was utilised as a carrier at a flow rate of $3 \mathrm{~mL} / \mathrm{min}$ in the column and the inlet split ratio was set at 20:1. The identification and quantitation of SCFAs were achieved by comparing the retention times and a peak area of unknown samples to that of commercial lipid standard (4-methylvaleric acid) as an internal control.

\section{Salmonella Typhimurium enumeration in organs}

At week 30 of flock age, the laying chickens were humanely euthanised by cervical dislocation and tissue pieces of various organs (spleen, liver, ovary, infundibulum/magnum, shell gland, jejunum and cecum) were aseptically collected into $1.5 \mathrm{~mL}$ Safe-Lock Eppendorf tubes containing stainless steel beads $0.5-2.0 \mathrm{~mm}$ and $500 \mu \mathrm{L}$ PBS. After weighing, tissues were homogenized using a bullet blender (Next Advance, USA) on full speed for 5-10 min. From the original tissue homogenates or serially diluted samples (cecum), $100 \mu \mathrm{L}$ was plated onto XLD agar and incubated overnight at $37^{\circ} \mathrm{C}$. Salmonella load was expressed as $\log _{10} \mathrm{CFU} / \mathrm{g}$ of tissue. A $100-\mu \mathrm{L}$ from the original homogenates was also enriched into $900 \mu \mathrm{L}$ buffered peptone water (BPW) and processed for Salmonella isolation through the enrichment method [40]. Putative Salmonella colonies on XLD were streaked on Brilliance Salmonella agar (BSA; Oxoid, Australia) plates and incubated overnight at $37^{\circ} \mathrm{C}$ for confirmation. Incubated plates were read as positive (scored as 1) or negative (scored as 0) for Salmonella based on the colony characteristics.

\section{Faecal DNA extraction and 16S rRNA sequencing}

Faecal DNA was extracted following the protocol of QIAamp FAST DNA Mini Kit with the inclusion of homogenisation step with glass beads (acid-washed $\leq 106 \mu \mathrm{m}$ and 425-600 $\mu \mathrm{m}$; Sigma Aldrich, Australia). The DNA quality was tested using a Nanodrop-1000 and the samples $(n=378)$ were submitted to the Ramaciotti Centre for Genomics (University of New South Wales, Australia) for 16S rRNA sequencing and generation of operational taxonomic units (OTUs) table. For generating $2 \times 300 \mathrm{bp}$ paired-end reads in Illumina, V3V4 region specific primer pair (341F: 5'-CCTACG GGNGGCWGCAG-3'; 805R: 5'-GACTACHVGGG TATCTAATCC-3') was used.
16S rRNA library preparation and Illumina sequencing The library was prepared using barcoding PCR in a 25$\mu \mathrm{L}$ reaction volume that contained $12.5 \mu \mathrm{L}$ KAPA HiFi HotStart Readymix (Kapa Biosystems), $1 \mu \mathrm{L}$ of each the primers, $1 \mu \mathrm{L}$ DNA template and $10.5 \mu \mathrm{L}$ PCR grade water. The thermal cycling conditions in SimpliAmp Thermal Cycler (Applied Biosystems) were: initial denaturation at $95^{\circ} \mathrm{C}$ for $3 \mathrm{~min}, 35$ cycles of denaturation at $95^{\circ} \mathrm{C}$ for $30 \mathrm{~s}$, annealing at $55^{\circ} \mathrm{C}$ for $30 \mathrm{~s}$ and elongation at $72^{\circ} \mathrm{C}$ for $30 \mathrm{~s}$, ending with a final elongation at $72^{\circ} \mathrm{C}$ for $5 \mathrm{~min}$. The PCR products were normalised and pooled using SequalPrep ${ }^{\mathrm{Tm}}$ Normalization Plate Kit (ThermoFisher Scientific, Australia) according to the manufacturer's instructions. The library was purified using Axygen AxyPrep Mag PCR Clean-Up Kit (Fisher biotec, Australia) as per the manufacturer's instructions. Concentration and quality of the pooled library were checked with Qubit and the library size on an Agilent 2200 TapeStation instrument. The Agencourt AMPure XP Bead Clean-up kit was used on the pool to reduce/remove the presence of primer dimers. The library pool was sequenced on Illumina MiSeq using a MiSeq Reagent Kit v3 with a $2 \times 300$ bp run format, using default run parameters including adaptor trimming. For these runs, custom primers were added to the reagent cartridge for Read1, Index and Read2.

\section{Microbial community data analysis for generation of OTU table}

Reads were processed with mothur (v1.39.5) [43] according to the MiSeq protocol [44]. Briefly, the reads were quality filtered and assigned to their respective samples. Samples were trimmed and only those with a length between 405 and 495 bp were retained. Samples with homopolymers longer than $8 \mathrm{bp}$ were removed. Chimeric sequences were removed using the chimera.vsearch script in mothur [45]. The sequences were aligned and classified against the SILVA reference alignment (v132) [45] and lineages not targeted by the primer pair (i.e. archaea, chloroplast, eukaryote, mitochondria and unknown) were removed. Sequences were grouped into OTUs based on $97 \%$ similarity using the OptiClust algorithm [46] and subsampled based on the sample with the lowest number of sequences, i.e. 25,556 sequences. Sequencing error was assessed using the ZymoBIOMICS Microbial Community Standard as control in each sequencing run. Interactive OTU plots were created with Krona [47] from the subsampled data. OTU richness plot was generated with the mothur_krona_XML.py script [48]. Diversity plots were generated by using the OTUsamples2krona.sh script [49] by providing a reformatted mothur biom file. 


\section{Statistical analysis}

The Salmonella Typhimurium load data in faeces $\left(\log _{10}\right.$ mMPN) and in organs (mean percent value) were analysed in Statview software (Version 5.0.1.0) by taking treatment and sampling time-point as main effects. Level of significance was determined by Fisher's protected least significant difference (PLSD) at $P<0.05$. For microbial community profiling, the OTU table was analysed in Calypso software [50] using one- and two-way ANOVA, redundancy analysis $(\mathrm{RDA}+)$, regression and diversity analyses. To remove the non-independence of relative microbial abundance, the data were transformed using the total sum normalisation (TSS) method [50, 51]. TSS normalises count data by dividing feature read counts by the total number of reads in each sample for obtaining relative abundance [50]. RDA is used to calculate complex association between microbial community composition and explanatory variables. In Calypso, false discovery rate (FDR) of $<0.05$ was used for level of significance between the treatment groups.

\section{Results}

\section{5 rRNA data and its quality}

The sequenced reads quality was as per Q30 standard and the average reads generated per sample were enough for genome alignment and the generation of OTU table for downstream analysis. The rarefaction analysis showed that the sequenced data covered well the diversity of the studied microbiota (Additional file 1: Figure S1). Overall, at phylum level, the microbial communities were clustered into Actinobacteria, Bacteroidetes, Cyanobacteria, Deferribacteres, Firmicutes, Proteobacteria, Synergistetes, Tenericutes and Verrucomicrobia (Additional file 2: Figure S2).

\section{Gut microbiota abundance and diversity are affected by Salmonella Typhimurium challenge}

To understand the effects of Salmonella Typhimurium on gut microbiota diversity and the abundance levels of different genera, the faecal microbiota data of the challenged laying chickens were analysed against the negative control group. Compared with the negative control, Salmonella challenge significantly $(\mathrm{FDR}<0.05)$ reduced the abundance of various bacterial genera that included Subdoligranulum, Shuttleworthia, Sellimonas, Ruminiclostridium_9, Intestinimonas, Gastranaerophilales_ge, Faecalibacterium, Enorma and Blautia (Fig. 1). The abundance levels of Oscillibacter, GCA900066225, Flavonifractor, Erysipelatoclostridium, Eisenbergiella, Caproiciproducens and Butyricicoccus were significantly increased in the Salmonella Typhimurium challenged group compared with the negative control group. The abundance of these genera was also visualised in individual samples of the same chickens obtained at

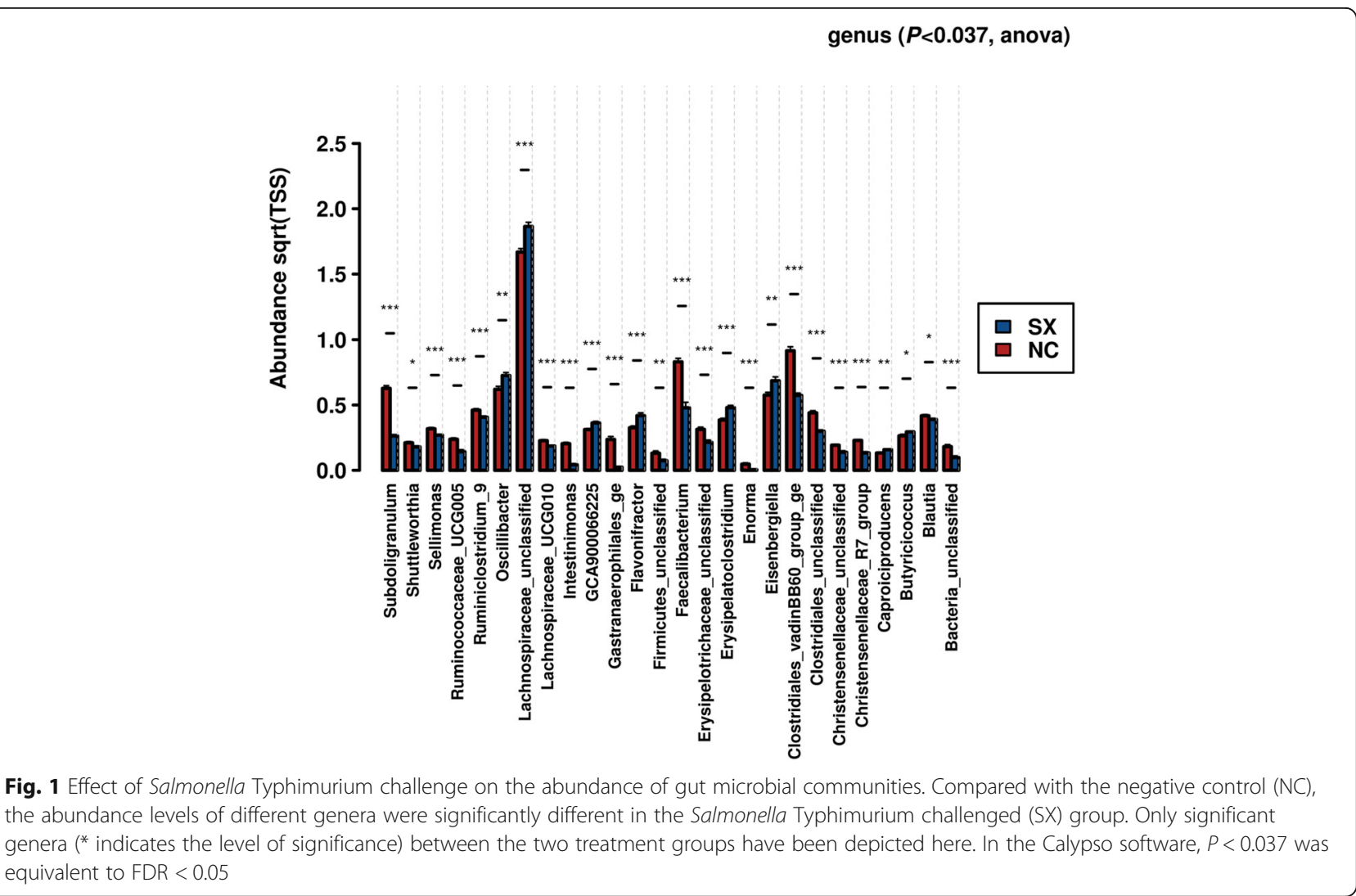


different sampling time-points (Additional file 3; Figure S3). The abundance of Bacteroides increased after week 8 postchallenge both in the negative control and Salmonella challenged groups.

A significant (FDR $<0.05)$ effect of sampling timepoint was observed on the abundance of multiple genera between the negative control and Salmonella Typhimurium challenged groups (Fig. 2; Additional file 4: Figure S4). The abundance levels of different genera varied differently with sampling time-points. Genera such as Subdoligranulum, Gastranaerophilales_ge, Intestinimonas, Ruminococcaceae_UCG005 and Sellimonas were consistently lower in abundance in the Salmonella Typhimurium challenged group at all sampling time-points. A correlation heatmap was used to understand the effects of the sampling time-points and Salmonella Typhimurium challenge on the abundance of individual genera of gut microbial communities. A clear pattern of representation of individual microbial communities at different time-points both in the negative control and Salmonella Typhimurium challenged groups shows that Salmonella challenge affected the abundance of multiple microbial genera (Fig. 3). Measured by redundancy analysis $(\mathrm{RDA}+)$, there was a significant $(P<0.05)$ effect of Salmonella challenge on the microbial community composition (Fig. 4a). The microbial alpha diversity (measured as Shannon index at genera level) was significantly different between the negative control (NC) and the Salmonella Typhimurium challenged (SX) group (Fig. 4b). The microbial diversity was significantly lower in the SX group across all the sampling time-points. Around week 4 post-challenge, two out of seven chickens were consistently negative for Salmonella Typhimurium. The gut microbiota analysis of the two Salmonella negative chickens showed a significantly higher abundance of Faecalibacterium, Erysipelotrichaceae_unclassified, Rikenellaceae_RC9_gut_group and Intestinimonas (Additional file 5: Figure S5).

To understand the effects of the probiotic on gut microbiota in the presence of Salmonella Typhimurium, the abundance of microbial genera was compared between the CPC and CPX and between the IPC and IPX groups. Compared with the CPC, Salmonella Typhimurium challenge significantly decreased the abundance levels of Acetanaerobacterium, Akkermansia, Anaerostipes, Bacteroides, Blautia, Eggerthella, Eisenbergiella, Enterococcus, EscherichiaShigella, Faecalibacterium, Lactobacillus, Melissococcus, Oscillibacter, Pediococcus, Ruminiclostridium_9, Ruminococcaceae_UCG014, Sellimonas, Subdoligranulum and Weissella, while increasing the abundance levels of Alistipes, Barnesiella, Bifidobacterium, Butyricimonas, Enorma, Intestinimonas, Megamonas, Parabacteroides, Paraprevotella, Parasutterella, Phascolarctobacterium and Sutterella (Additional file 6: Figure S6).

The microbial community composition of the CPC was significantly separated from the CPX group (Additional file 7: Figure S7a). The microbial diversity was significantly lower in the CPC compared with the CPX across all the sampling time-points (Additional file 7:

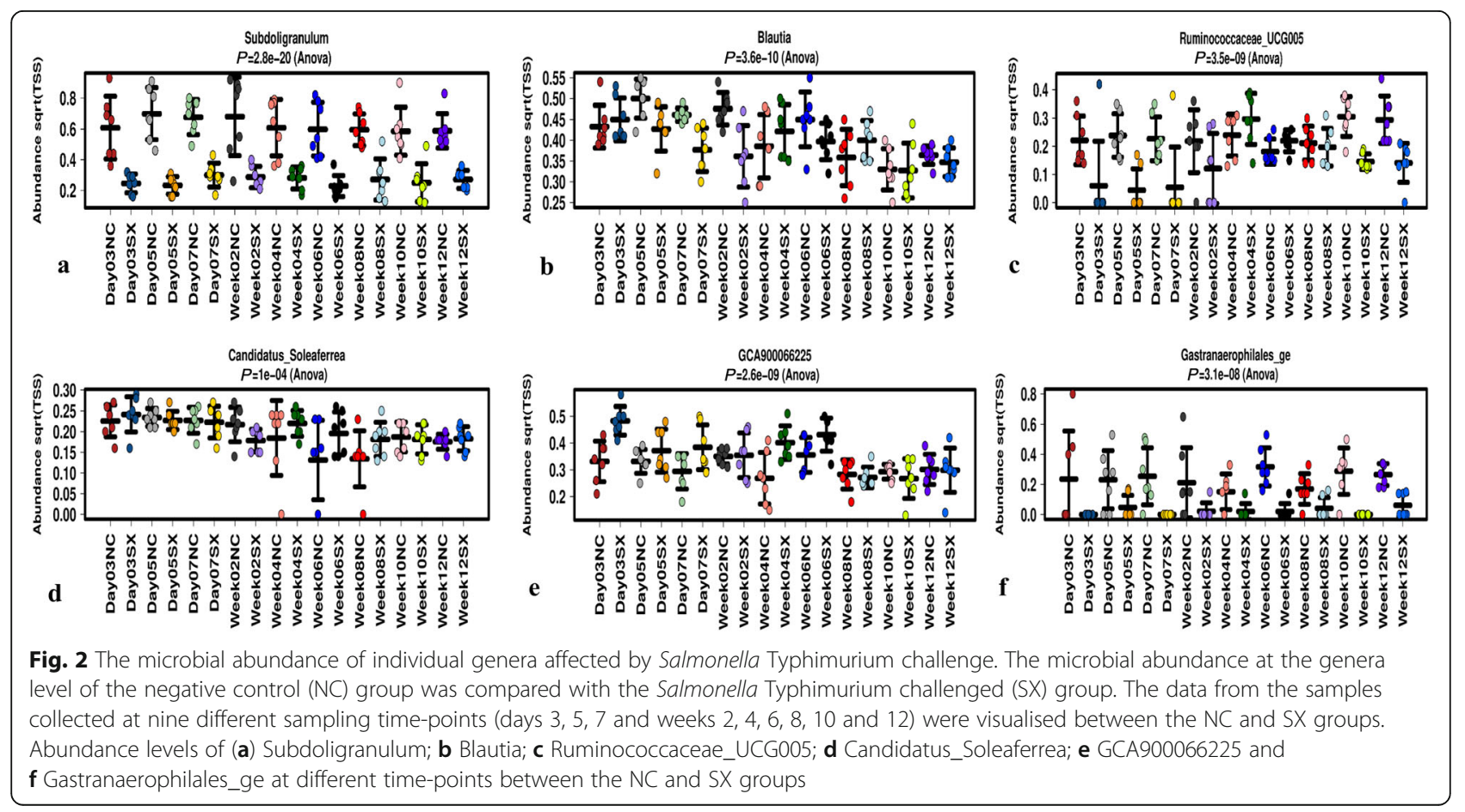



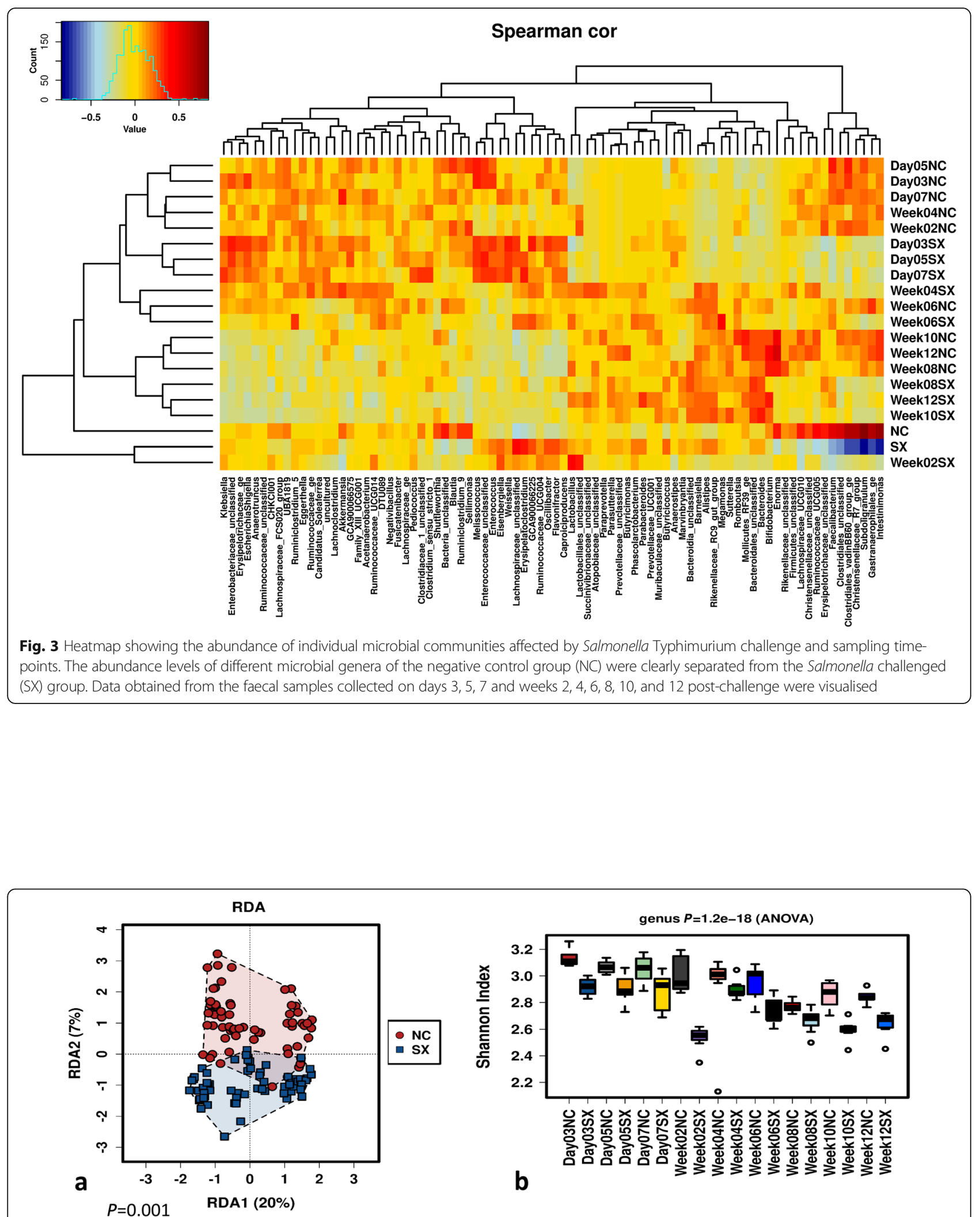

Fig. 4 Microbial community composition and diversity affected by Salmonella Typhimurium challenge. a Microbial community composition between the negative control (NC) and Salmonella Typhimurium challenged (SX) groups. b Microbial diversity between the NC and SX at different time-points (days 3, 5, 7 and weeks 2, 4, 6, 8, 10 and 12) post-challenge 
Figure S7b). The abundance levels of microbial genera in the IPC and IPX treatment groups were comparable to the CPC and CPX treatment groups, but there were fewer genera significantly affected between the two treatment groups of IPC and IPX (Additional file 8: Figure S8). The microbial community composition of the IPX group was clearly separated from the IPC group and diversity of the IPX treatment group was significantly higher than the IPC in some of the sampling time-points (Additional file 9: Figure S9a, b).

To determine the effects of probiotic supplementation on microbial abundance and diversity, data were analysed and compared between the negative control and the probiotic supplemented control groups (excluding Salmonella Typhimurium challenge). Compared to the negative control, the continuous supplementation of the probiotic decreased the diversity of microbiota (Additional file 10: Figure S10a) and the abundance of Eisenbergiella, EscherichiaShigella, Blautia, Flavonifractor and Subdoligranulum (Additional file 10: Figure S10b). Compared with the negative control, the intermittent supplementation of probiotic decreased the diversity of microbiota
(Additional file 11: Figure S11a) and the abundance of microbial genera, such as Faecalibacterium, EscherichiaShigella, Blautia, Sellimonas and Subdoligranulum (Additional file 11: Figure 11b).

\section{Gut microbiota displaced by Salmonella Typhimurium was restored by Bacillus based probiotic supplementation}

To understand the effects of the Bacillus based probiotic in restoring the gut microbial community abundance, we analysed the data obtained from the chickens continuously or intermittently fed with probiotic supplement and challenged with Salmonella Typhimurium or left as probiotic controls. The data were analysed against each respective treatment groups. The abundance levels of microbial genera that were significantly decreased or increased by the Salmonella Typhimurium challenge (SX) compared with the negative control (NC) group, were assessed for the effects of the probiotic. Probiotic supplementation restored (FDR >0.05) the abundance levels of microbial genera, such as Bacteria_unclassified, Christensenellaceae_R7_group, Christensenellaceae_ unclassifed, Lachnospiraceae_UCG010, Ruminiclostridium_9, Erysipelotrichaceae_unclassified, Firmicutes_

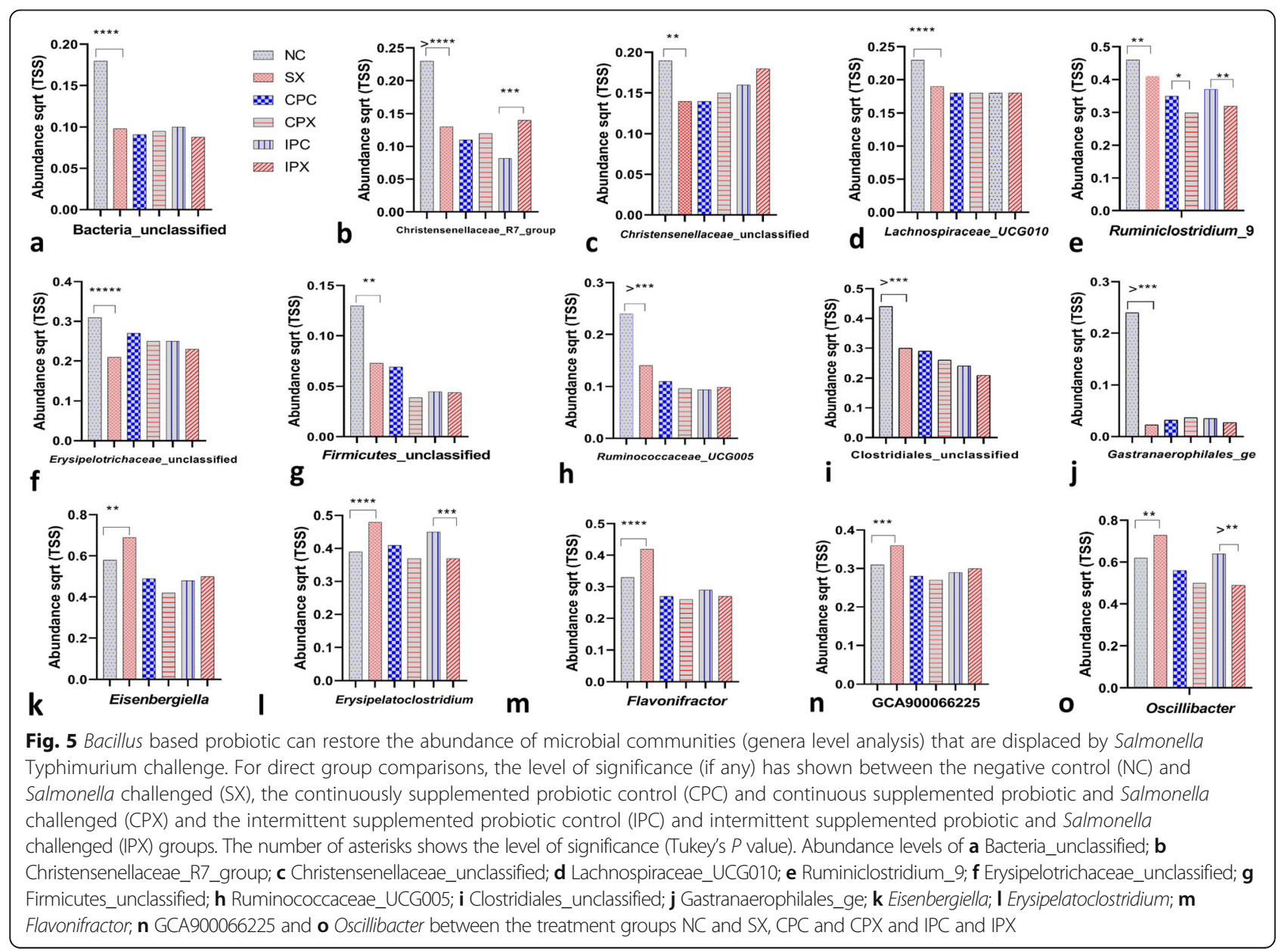


unclassified, Ruminococcaceae_UCG005, Clostridiales_ unclassified and Gastranaerophilales_ge (Fig. 5 a-j). Compared with the negative control, Salmonella challenge significantly increased the abundance of Eisenbergiella, Erysipelatoclostridium, Flavonifractor, GCA900066225 and Oscillibacter (Fig. 5 k-o). When the effects of the continuously and intermittently supplemented Bacillus based probiotic on the restoration of the abundance of these microbial communities were assessed, the data showed that the continuously and intermittently supplemented probiotic restored microbiota with clearer effects observed for the continuously supplemented probiotic (Fig. 5 a-o).

\section{Microbial abundance affected by Salmonella Typhimurium} was different in the presence of probiotic

The effect of the Bacillus based probiotic on the abundance of microbial communities at genera level in the presence and absence of Salmonella Typhimurium challenge was also assessed. Compared with the probiotic supplemented control groups, Salmonella challenge significantly reduced the abundance of Acetanaerobacterium, Pediococcus, Anaerostipes, Eggerthella, Bacteroides and Lactobacillus in the probiotic supplemented and Salmonella Typhimurium challenged groups (Fig. 6a-f). This effect was highly significant for the continuously supplemented probiotic compared with the intermittently supplemented probiotic group (Fig. 6a-f). Interestingly, the abundance of Butyricimonas, Anaerotruncus, Barnesiella, Megamonas, Parabacteroides, Paraprevotella, Parasutterella, Alistipes, Phascolarctobacterium and Sutterella was significantly higher in the probiotic supplemented and Salmonella challenged groups compared with the probiotic supplemented control groups (Fig. 6g-p). The abundance of these microbial communities was not significantly different between the negative control and Salmonella challenged groups (Fig. 6a-p).

\section{Gut microbiota drives Salmonella Typhimurium load}

To understand the interaction of Salmonella Typhimurium load with the gut microbiota at individual genera level, a regression analysis was performed on the $\log _{10}$ mMPN values of individual birds against each of the genera in the Salmonella challenged (SX) group. The load of Salmonella Typhimurium in the gut significantly $(P<0.05)$ affected the abundance of different microbial genera. The abundance of

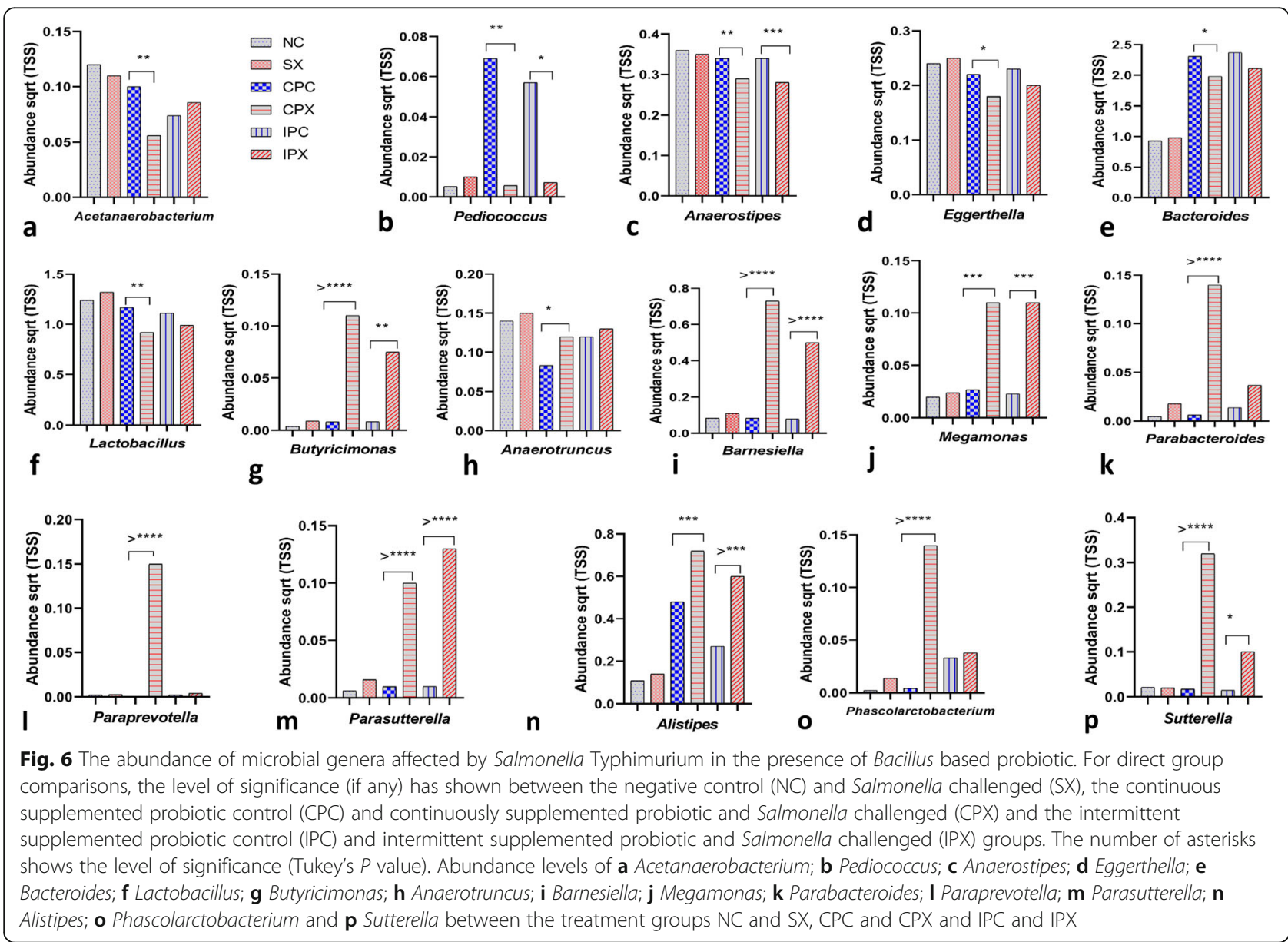


Table 1 Correlation of Salmonella Typhimurium load with abundance of microbial genera in faeces

\begin{tabular}{|c|c|c|c|c|c|}
\hline Microbe & $R$ value & $P$ value & Microbe & $R$ value & $P$ value \\
\hline Alistipes & -0.341 & $1.60 \mathrm{E}-06$ & Prevotellaceae_UCG001 & -0.323 & $5.70 \mathrm{E}-06$ \\
\hline Atopobiaceae_unclassified & -0.442 & $1.80 \mathrm{E}-10$ & Prevotellaceae_unclassified & -0.297 & 3.30E-05 \\
\hline Bacteroidales_unclassified & -0.279 & 1.00E-04 & Rikenellaceae_RC9-gut-group & -0.258 & 3.30E-04 \\
\hline Bifidobacterium & -0.531 & $3.70 \mathrm{E}-10$ & Acetanaerobacterium & 0.181 & 0.013 \\
\hline Barnesiella & -0.433 & 5.00E-10 & Akkermansia & 0.212 & 0.0034 \\
\hline Butyricimonas & -0.233 & $1.30 \mathrm{E}-03$ & Anaerostipes & 0.153 & 0.036 \\
\hline Christensenellaceae_R7_group & -0.146 & 4.40E-02 & Anaerotruncus & 0.179 & 0.014 \\
\hline Clostridiales_vadinB860_group_ge & -0.346 & $1.10 \mathrm{E}-06$ & Blautia & 0.258 & 0.00033 \\
\hline Enorma & -0.403 & $9.10 \mathrm{E}-09$ & Caproiciproducens & 0.192 & 0.0082 \\
\hline Faecalibacterium & -0.213 & 3.30E-03 & Clostridiaceae_1_unclassified & 0.299 & $2.90 \mathrm{E}-05$ \\
\hline Family_XIII_UCG001 & -0.318 & 8.10E-06 & Clostridium_sensu_stricto_1 & 0.25 & 0.00051 \\
\hline Intestinimonas & -0.301 & $2.50 \mathrm{E}-05$ & Eggerthella & 0.39 & $2.90 \mathrm{E}-08$ \\
\hline Lactobacillus & -0.384 & 4.80E-08 & Eisenbergiella & 0.416 & 2.70E-09 \\
\hline Megamonas & -0.309 & $2.60 \mathrm{E}-05$ & Enterococcaceae_unclassified & 0.397 & 1.60E-08 \\
\hline Negativibacillus & -0.168 & 2.10E-02 & Erysipelatoclostridium & 0.391 & 2.70E-08 \\
\hline Parabacteroides & -0.301 & $2.60 \mathrm{E}-05$ & Erysipelotrichaceae_ge & 0.29 & $5.00 \mathrm{E}-05$ \\
\hline Paraprevotella & -0.242 & 7.90E-04 & EscherichiaShigella & 0.542 & $8.90 \mathrm{E}-16$ \\
\hline Parasutterella & -0.304 & $2.10 \mathrm{E}-05$ & Flavonifractor & 0.268 & $1.90 \mathrm{E}-04$ \\
\hline Phascolarctobacterium & -0.194 & 7.50E-03 & Fusicatenibacter & 0.21 & $3.70 \mathrm{E}-03$ \\
\hline Romboutsia & -0.304 & 2.10E-05 & GCA900066575 & 0.422 & 1.50E-09 \\
\hline Sutterella & -0.377 & $9.20 \mathrm{E}-08$ & Lachnospiraceae_unclassified & 0.267 & 0.00021 \\
\hline Succinivibrionaceae_unclassified & -0.26 & 3.00E-04 & Melissococcus & 0.252 & 4.60E-04 \\
\hline Ruminococcaceae_UCG005 & -0.355 & $5.50 \mathrm{E}-07$ & Pediococcus & 0.22 & $2.40 \mathrm{E}-03$ \\
\hline Rikenellaceae_unclassified & -0.318 & 8.40E-06 & Ruminiclostridium_5 & 0.279 & 1.00E-04 \\
\hline Bacteroidia_unclassified & -0.186 & $1.00 \mathrm{E}-02$ & Ruminococcaceae_unclassified & 0.146 & 4.60E-02 \\
\hline Mollicutes_RF39_ge & -0.281 & 8.90E-05 & Sellimonas & 0.333 & $2.90 \mathrm{E}-06$ \\
\hline Muribaculaceae_unclassified & -0.309 & $1.80 \mathrm{E}-05$ & Weissella & 0.352 & $6.70 \mathrm{E}-07$ \\
\hline
\end{tabular}

The faecal load of Salmonella Typhimurium (in $\log _{10} \mathrm{mMPN}$ ) was regressed against the abundance of individual genera of microbiota. Minus (-) sign shows negative correlation

30 microbial genera showed a significant weak negative correlation with the Salmonella Typhimurium load in the gut (Table 1). These genera included important gut resident microbiota members such as Lactobacillus, Megamonas, Enorma, Barnesiella, Butyricimonas, Faecalibacterium, Intestinimonas and Parabacteroides. The abundance of 24 microbial genera showed a significant weak positive correlation with the Salmonella Typhimurium load in the gut (Table 1). These microbial communities included genera such as Acetanaerobacterium, Akkermansia, Anaerostipes, Blautia, Eggerthella, Pediococcus and EscherichiaShigella.

\section{Short chain fatty acids quantification from faeces}

The levels of acetate, butyrate and propionate were significantly $(P<0.05)$ affected over time following Salmonella Typhimurium infection (Fig. 7). Among the treatment groups, the levels of acetate and butyrate were significantly higher in the continuously supplemented probiotic control (CPC) compared with the continuously supplemented probiotic and Salmonella Typhimurium challenged (CPX) group. However, within each treatment group, at each sampling time-point, there was no significant $(P>0.05)$ difference in the acetate content of the faeces (Fig. 7a). Within each treatment group, the level of butyrate in the faeces was significantly higher in the CPC and intermittent supplemented probiotic control (IPC) groups compared with the CPX and the intermittent supplemented probiotic and Salmonella Typhimurium challenged (IPX) groups. Within each treatment group, the level of butyrate in the faeces at week 1, 4 and 8 post-challenge was significantly higher in the CPC compared with the IPX group (Fig. 7b). The propionate level was significantly affected by the sampling time-point post Salmonella Typhimurium challenge but was not consistent with the levels of acetate and butyrate (Fig. 7c). Within each treatment group, the level of propionate in 


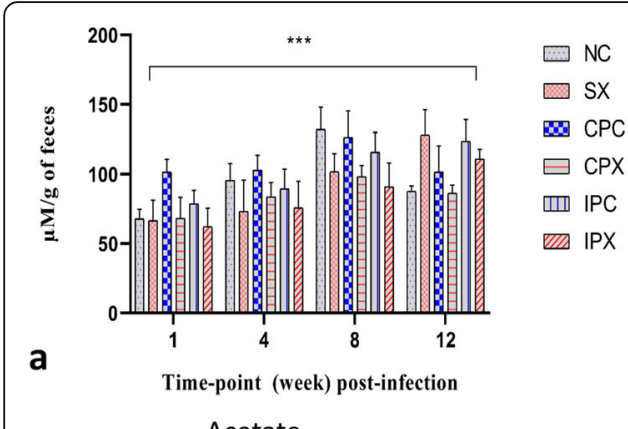

Acetate
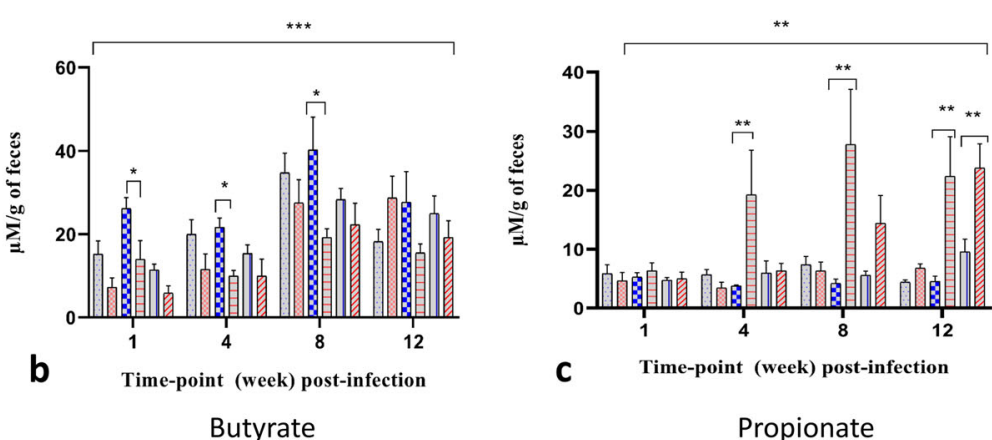

Fig. 7 Short-chain fatty acids levels in the faeces of chickens fed with probiotic and challenged with Salmonella Typhimurium. The respective treatment groups were compared with each other at each sampling time-point of Salmonella Typhimurium post-challenge. a Acetate, $\mathbf{b}$ butyrate and $\mathbf{c}$ propionate levels in faeces at different sampling time-points (weeks 1, 4, 8 and 12 post-challenge). Bar (with asterisks) across the individual graph shows a significant effect of treatment group on short-chain fatty acids production. NC is negative control; SX is Salmonella challenge; CPX is continuous probiotic supplemented and Salmonella challenge; CPC is continuous probiotic supplemented control; IPX is intermittent probiotic supplemented and Salmonella challenge; IPC is intermittent probiotic control

the faeces was significantly lower in the CPC and IPC compared with the CPX and IPX groups.

\section{Effects of probiotic supplementation on Salmonella Typhimurium load in faeces and organs}

To understand the effects of gut microbiota modulation through the probiotic on Salmonella Typhimurium shedding levels in faeces, an mMPN method $\left(\log _{10}\right)$ was performed. Faeces from the negative and probiotic control groups were negative for Salmonella. Irrespective of the probiotic supplementation, some chickens from the Salmonella challenged groups turned negative for Salmonella Typhimurium load in faeces around week 4 post-challenge. However, not all of these chickens were consistently negative for faecal load of Salmonella at different sampling time-points. A significant effect of timepoint and treatment was observed on the shedding level of Salmonella Typhimurium in the faeces (Fig. 8a, b). Within each sampling time-point, the continuously supplemented probiotic and Salmonella Typhimurium challenged group (CPX) showed a significantly lower bacterial load compared with the intermittent supplemented probiotic and Salmonella Typhimurium challenged group (IPX) at week 8 post-challenge (Fig. 8a). Overall, the load of Salmonella Typhimurium was significantly lower in the CPX compared with the Salmonella challenged (SX) and IPX groups (Fig. 8b).

The load of Salmonella Typhimurium in organs was determined at the point of termination of the experiment (week 30 of flock age). Salmonella was not recovered from various organs collected from the negative and probiotic control groups. For the Salmonella Typhimurium challenged groups, organ homogenates directly plated on XLD and BSA media were negative; however, some samples turned positive when an enrichment method was followed. Therefore, the load of Salmonella Typhimurium in organs was expressed as mean percent value per treatment group. The mean percent value of Salmonella Typhimurium for caecum was significantly lower in the CPX compared with the SX group (Fig. 8c) The mean percent value of Salmonella Typhimurium for shell gland was significantly lower in the CPX and IPX compared with the SX group (Fig. 8h). Salmonella Typhimurium was not recovered from the cecum, jejunum, liver, magnum/infundibulum and shell gland of the CPX group (Fig. 8c, d, e, g, h). Similarly, Salmonella Typhimurium was not recovered from the liver, magnum/infundibulum and shell gland of the IPX group (Fig. 8e, g, h). No Salmonella was isolated from the internal contents of the eggs. Shell wash samples positive for Salmonella through the enrichment method showed no measurable load by the mMPN method.

\section{Discussion}

The main objectives of this study were to understand the dynamics of the gut microbiota in Salmonella Typhimurium infected laying chickens, and to study the effects of continuous and intermittent feeding of probiotic on Salmonella Typhimurium shedding. A balanced gut microbiota can resist pathogen colonisation and subsequent clearance from the gut [52]. In this study, we reared Salmonella spp. free laying chickens to understand the true effects of this pathogen on gut microbiota displacement as other species of Salmonella, if already colonised in gut, can significantly influence the shedding of Salmonella Typhimurium. The results showed that both the Salmonella Typhimurium and the Bacillus based probiotic significantly affected the composition and diversity of the gut microbial communities. The data also showed that continuous supplementation of the $\mathrm{Ba}$ cillus based probiotic reduced the load of Salmonella Typhimurium in the faeces (overall) and in organs tested at the end of the experiment. The decrease in abundance 


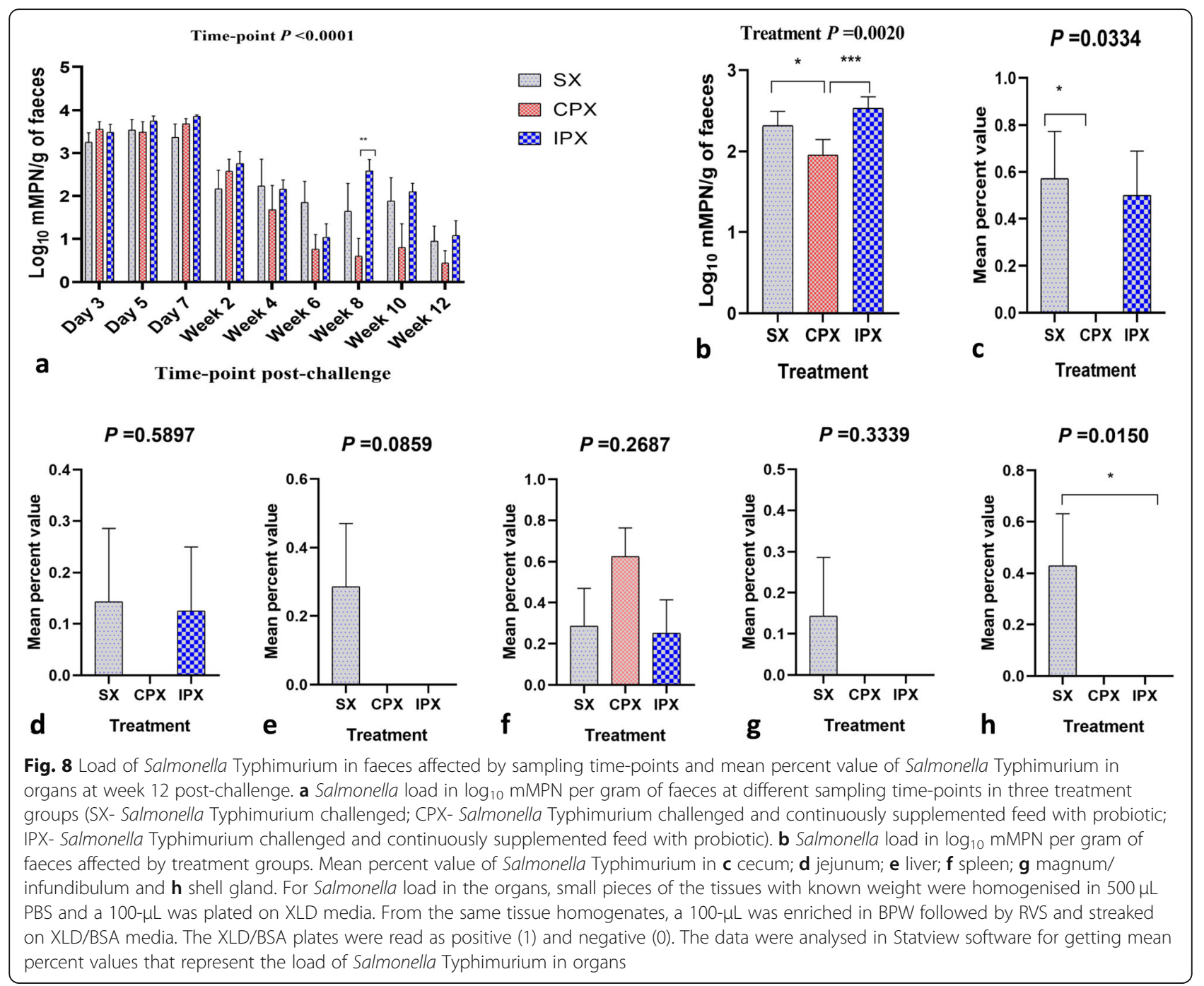

levels of Eisenbergiella, EscherichiaShigella, Blautia, Flavonifractor and Subdoligranulum by the probiotic supplementation shows that the Bacillus based probiotic has the potential to affect gut microbial abundance. Of the reduced microbial genera, Escherichia and Shigella have the potential to cause infection in certain conditions, while other genera such as Blautia, Flavonifractor and Subdoligranulum are vital for gut health. The probiotic supplementation also increased the abundance levels of good bacteria, such as Bacteroides and Alistipes. Further investigation is necessary to determine the effects of the decreased abundance of the above mentioned microbial genera on the host gut.

In chickens, the composition of gut microbiota varies considerably with bird age, with more complex microbiota present in older birds [53]. Salmonella Typhimurium induces inflammation of intestinal epithelia [37] and displacement of gut microbiota in laying chicks [54]. However, the long-term effects of Salmonella Typhimurium on the gut microbiota in laying chickens have not been investigated. To understand the role of Salmonella Typhimurium colonisation on the abundance and diversity of gut microbiota, the microbial communities of the faeces of individual birds collected at nine different timepoints post-challenge from the Salmonella negative control and Salmonella challenged groups were compared. Overall, the Salmonella Typhimurium infection reduced the abundance of many bacterial genera including Blautia, Bacteria_unclassified, Christensenellaceae R7_group, Enorma, Faecalibacterium, Christensenellaceae_unclassifed, Lachnospiraceae_UCG010, Ruminiclostridium_9, Subdoligranulum and Firmicutes_unclassified. This shows that not all members of the gut microbiota have the potential to compete with the Salmonella Typhimurium. Most of these bacterial genera play a vital role in maintaining gut health through the production of organic acids and vitamins. For example, Christensenellaceae contains bacteria that secrete $\beta$-glucosidase, $\beta$ - 
galactosidase and $\alpha$-arabinosidase and therefore help in polysaccharide digestion [55]. As Salmonella lacks the enzyme, $\beta 1-4$ linkage required for polysaccharide fermentation, Christensenellaceae could ferment it. However, Salmonella Typhimurium challenge reduces the abundance of Christensenellaceae and Lachnospiraceae [54]. Therefore, Salmonella infection could lead to the interruption of the Christensenellaceae based polysaccharide fermentation. Ruminiclostridium_9 and Ruminococcaceae_UCG005 are members of Ruminococcaceae that are common gut microbes involved in the breakdown of complex carbohydrates. A decreased abundance of Erysipelotrichaceae was observed in Crohn's disease [56]. Some species in Clostridiales degrade a variety of fibre and have been identified as producing propionate, acetate and butyrate. Gastranaerophilales_ge obtains its energy by obligate fermentation resulting in the production of organic acids in the gut. A previous study suggested that Salmonella Enteritidis reduced the abundance level of Faecalibacterium in the chicken gut [57]. The functions of Faecalibacterium in the chicken gut are not well characterised; however, it is one of the most abundant resident gut microbes in a human gut [58]. In the current study, the lower abundance levels of the above mentioned microbial communities show that Salmonella Typhimurium establishes its niche in the gut at the expense of displacing these bacterial communities leading to Salmonella driven dysbiosis. In the current study, the increased abundance of Faecalibacterium in the gut of hens that turned negative for Salmonella suggest its potential role to be characterised as a probiotic candidate for gut health.

Compared with the negative control group, Salmonella Typhimurium challenge increased the abundance of Eisenbergiella, Erysipelatoclostridium, Flavonifractor, GCA900066225 and Oscillibacter. Eisenbergiella is a rod-shaped, non-proteolytic, non-motile, anaerobic bacteria in the Lachnospiraceae that produces succinate, lactate, butyrate and acetate during fermentation [59]. Erysipelatoclostridium is a part of normal gut microbiota but could become an opportunistic pathogen and has been identified as a gut microbiota biomarker in human patients suffering from Crohn's disease and Clostridium difficile infection [60]. In the current study, the nonsignificant difference in the abundance levels of Eisenbergiella, Flavonifractor, GCA900066225, Oscillibacter and Erysipelatoclostridium between the continuously supplemented probiotic control and the continuously supplemented probiotic and Salmonella Typhimurium challenged groups shows the positive effect of the probiotic on gut microbiota. These results are further supported by the positive correlation of the abundance levels of the above-mentioned genera with Salmonella load in the gut.
The regression analysis of the Salmonella Typhimurium load (measured as $\log _{10} \mathrm{mMPN} / \mathrm{g}$ of faeces) against the abundance of gut microbial genera showed that more genera were negatively affected by the Salmonella Typhimurium infection. This indicates that, as the Salmonella Typhimurium load decreased over time, these microbial genera had the potential to restore normal abundance. The negatively correlated genera, such as Lactobacillus, Megamonas, Negativibacillus, Parabacteroides, Paraprevotella, Parasutterella, Phascolarctobacterium, Romboutsia, Bifidobacterium, Butyricimonas, Barnesiella, Faecalibacterium and Intestinimonas perform vital functions ranging from vitamin synthesis to organic acid production. Megamonas contains a gene cluster that encodes secreted cellobiose phosphotransferase system, endo-glucanases and 6phospho-beta-glucocidase that potentially degrade nonstarch polysaccharides to cellobiose in the chicken gut [61]. Negativibacillus belongs to Ruminococcaceae with no known functions. Parabacteroides improves host metabolism through the production of succinate and secondary bile acids in the gut as shown in mice [62]; however, its functions in chickens have not been investigated. For propionate production, Parabacteroides, Alistipes and Paraprevotella express cobalamin-binding methylmalonylCoA mutase and/or methylmalonyl-CoA epimerase [63]. In Firmicutes, Phascolarctobacterium, Megamonas and Blautia produce propionate through epimerase, decarboxylase and methylmalonyl-CoA mutase pathways [63]. Faecalibacterium, Subdoligranulum, and Phascolarctobacterium produce butyrate through acetyl/propionyl-CoA carboxylase pathway. In the current study, the reduced abundance of the useful microbial genera by the Salmonella Typhimurium challenge would have affected their normal functions vital for maintaining gut health through fermentation. Moreover, most of these microbial communities were positively influenced when the probiotic was supplemented in the diet. The effects of the probiotic on the abundance at the genera level were clearer in the continuously supplemented, rather than the intermittent supplemented group. For example, the continuously supplemented probiotic restored the abundance of Christensenellaceae_R7_group, Erysipelatoclostridium and Oscillibacter, while the intermittent supplementation merely improved it compared with the Salmonella challenged groups. This shows that the continuous supplementation of the probiotic produced better results.

On the other hand, the increased load of Salmonella Typhimurium favoured a large number of microbial communities of the gut microbiota by increasing their abundance. The bacterial genera that were positively correlated with the Salmonella Typhimurium load included Flavonifractor, Akkermansia, Anaerostipes, Blautia, Caproiciproducens, Eggerthella, Eisenbergiella, Erysipelatoclostridium, Melisococcus, Pediococcus, Ruminiclostridium_5, 
Sellimonas, Weissella and some unclassified genera. Although these genera are part of normal gut microbiota, some of them can become opportunistic pathogens causing dysbiosis and subsequent infections. The precise molecular mechanisms underlying how Salmonella Typhimurium causes the increased abundance of these genera are not known; however, in this study, we showed that the Salmonella driven dysbiosis favours a large number of resident gut microbiota to increase in abundance thereby affecting the abundance of other resident gut microbial community members. Flavonifractor is a member of resident gut microbiota but has been shown to cause infection in an immunocompromised patient [64]. The precise role of Flavonifractor in the dysbiosed gut of chickens needs to be investigated.

In the current study, the levels of acetate, butyrate and propionate in faeces were quantified at week $1,4,8$ and 12 post-challenge to understand the effects of the probiotic treatment in Salmonella challenged or nonchallenged hens. The higher level of butyrate in response to the supplementation of the probiotic shows that the probiotic treatment increased its production, while the Salmonella infection decreased it possibly due the displaced microbial communities. The microbiota produced gut metabolites such as acetate, butyrate and propionate. These metabolites play an important role in gut health ranging from the provision of energy to host enterocytes and regulation of the immune system [65]. The propionate level was higher in Salmonella challenged and probiotic supplemented groups compared to the probiotic control groups. It seems that certain organic acid producing genera that increased in abundance in response to Salmonella infection may have produced propionate. However, this needs further investigation.

The inconsistency in the Salmonella positive faecal samples from the infected groups with the Salmonella status of the ceca (at point of termination of the experiment) might highlight the importance of Salmonella persistence in other parts of the gut, such as colon, which requires further investigation. Irrespective of the probiotic supplementation status, the faeces of some Salmonella challenged chickens turned negative for Salmonella around week 4 post-challenge but were inconsistent in shedding profile. However, around week 8 post-challenge, more hens turned negative for Salmonella Typhimurium shedding in the faeces in the continuous supplemented probiotic $(n=5)$ compared with the intermittent supplemented probiotic $(n=2)$ and Salmonella challenged $(\mathrm{n}=3)$ groups. This shows that the Salmonella challenged chickens could harbour the bacteria in the gut for intermittent shedding. Probiotic treatment can reduce the level of shedding but continuous or intermittent feeding of probiotics does not eliminate the pathogen.

\section{Conclusions}

Salmonella Typhimurium affects the microbial abundance of certain genera that play a role in maintaining a healthy gut. Microbial genera that are increased in abundance in the Salmonella populated gut might play a role either in the Salmonella driven dysbiosis or in maintaining a normal gut function. The displaced gut microbiota can be partly restored by supplementing the feed with a Bacillus based probiotic, thus lowering the mean load of Salmonella in faeces.

\section{Supplementary information}

Supplementary information accompanies this paper at https://doi.org/10. 1186/s40104-020-0433-7.

Additional file 1: Figure S1. Rarefaction analysis of OTUs showing the quality of the reads generated from DNA obtained from chicken faeces. The flatten curves towards right show that the underlying microbial communities were well covered by the sequenced data. NC is negative control; SX is Salmonella challenge; CPX is continuous probiotic supplemented and Salmonella challenge; CPC is continuous probiotic supplemented control; IPX is intermittent probiotic supplemented and Salmonella challenge; IPC is intermittent probiotic control.

Additional file 2: Figure S2. Abundance of microbial communities at phylum level in faeces. Data for all the treatment groups were mapped in Calypso software to get the abundance of different phyla.

Additional file 3: Figure S3. Abundance of microbial communities at genera level in faecal samples of individual chickens in the negative control and Salmonella challenged chickens sampled at different timepoints (days 3, 5, 7 and weeks 2, 4, 6, 8, 10 and 12 post-challenge). The genus bar is based on sampling time-points post-challenge.

Additional file 4; Figure S4. Faecal microbial abundance affected by Salmonella Typhimurium challenge at different sampling time-points in laying chickens. Panel labels (a-d) show the effect of Salmonella on individual bacterial genera. NC is negative control, SX is Salmonella challenged. Data from the faecal samples collected at days 3, 5, 7 and weeks 2, 4, 6, 8, 10 and 12 post-challenge were used for comparison between the two treatment groups (NC and SX).

Additional file 5: Figure S5. Microbial genera abundance of Salmonella turned negative chickens. The abundance level of the Salmonella turned negative chickens $(n=2)$ was compared with consistently Salmonella shedding chickens $(n=5)$ and negative control groups $(n=7)$.

Additional file 6: Figure S6. Microbial genera abundance affected by Salmonella Typhimurium challenge and continuous supplementation of probiotic. The microbial abundance at genera level of the continuous supplemented probiotic control (CPC) group was compared with the continuous supplemented probiotic and Salmonella Typhimurium challenged (CPX) group. Data from the faecal samples collected at nine different sampling time-points (days 3, 5, 7 and weeks 2, 4, 6, 8, 10 and 12) post-challenge were analysed for comparison between the two treatment groups (CPC and CPX).

Additional file 7: Figure S7. Microbial community composition and diversity affected by Salmonella Typhimurium and continuous supplementation of probiotic. (a) Microbial community composition between the continuous supplemented probiotic control (CPC) and the continuous supplemented probiotic and Salmonella Typhimurium challenged (CPX) groups. (b) Microbial diversity between the CPC and CPX at different time-points (days 3, 5, 7 and weeks 2, 4, 6, 8, 10 and 12) post-challenge. Data from the faecal samples collected at days 3, 5, 7 and weeks 2, 4, 6, 8, 10 and 12 post-challenge were used for the comparison between the two treatment (CPC and CPX) groups.

Additional file 8: Figure S8. Microbial abundance of individual genera affected by Salmonella Typhimurium challenge and intermittent supplementation of probiotic. The microbial abundance at genera level 
of the intermittent supplemented probiotic control (IPC) group was compared with the intermittent supplemented probiotic and Salmonella Typhimurium challenged (IPX) group. Data from the faecal samples collected at nine different sampling time-points (days 3, 5, 7 and weeks 2, 4, 6, 8, 10 and 12) post-challenge were analysed for comparison between the two treatment groups (IPC and IPX).

Additional file 9: Figure S9. Microbial community composition and diversity affected by Salmonella Typhimurium and intermittent supplementation of probiotic. (a) Microbial community composition between the intermittent supplemented probiotic control (IPC) and the intermittent supplemented probiotic and Salmonella Typhimurium challenged group (IPX). (b) Microbial diversity between the IPC and IPX at different time-points (days 3, 5, 7 and weeks 2, 4, 6, 8, 10 and 12) postchallenge. Data from the faecal samples collected at days 3, 5, 7 and weeks $2,4,6,8,10$ and 12 post-challenge were analysed for comparison between the two treatment groups (IPC and IPX).

Additional file 10: Figure S10. Microbiota diversity and abundance of microbial genera affected by continuous supplementation of probiotic. (a) Overall diversity of faecal microbiota. (b) Abundance of faecal microbial genera. For determining the effects of the probiotic on the diversity of gut microbiota and abundance levels of individual microbial genera, the negative control (NC) group was compared with the continuous supplemented probiotic (CPC) group (excluding Salmonella Typhimurium challenge).

Additional file 11: Figure S11. Microbiota diversity and abundance of microbial genera affected by intermittent supplementation of probiotic. (a) Overall diversity of faecal microbiota. (b) Abundance of faecal microbial genera. For determining the effects of the probiotic on the diversity of gut microbiota and abundance levels of individual microbial genera, the negative control (NC) group was compared with the intermittent supplemented probiotic (IPC) group (excluding Salmonella Typhimurium challenge).

\section{Abbreviations}

BPW: Buffered peptone water; BSA: Brilliance Salmonella agar; FDR: False discovery rate; LB: Luria-Bertani; mMPN: Miniaturised most probable number; OTUs: Operational taxonomy units; PBS: Phosphate buffered saline; RDA + : Redundancy analysis; SCFAs: Short chain fatty acids; TSS: Total sum scaling or total sum normalisation; XLD: Xylose lysine deoxycholate

\section{Acknowledgements}

Help from Andrea McWhorter and Nitish Narendra Joat in chicken dissection is acknowledged. Andrea is a Research Fellow and Nitish is a PhD student at The University of Adelaide, Australia. Critical reading of the manuscript by Andrea McWhorter and Juliet Roberts (University of New England, Australia) is also acknowledged.

\section{Authors' contributions}

KKC and SK contributed to the study design, animal trials, samples processing and critical revision of the manuscript. SK and KKC performed the animal trials, SK processed the samples, analysed the data and drafted the manuscript. Both the authors approved the manuscript for publication.

\section{Funding}

This research was financially supported by Australian Eggs under the grant number 1FS802UA.

\section{Availability of data and materials}

The 165 rRNA sequence data are available from the NCBI SRA under the BioProject accession number PRJNA561675.

\section{Ethics approval and consent to participate}

The experimental setup was approved by the University of Adelaide, Animal Ethics Committee under Approval Number S-2017-080. The protocol was carried out in accordance with the guidelines -specified in Australian Code for the Care and Use of Animals for Scientific Purposes 8th edition 2013.

\section{Consent for publication}

Not applicable.

\section{Competing interests}

The authors declare that they have no competing interests.

Received: 2 November 2019 Accepted: 2 February 2020

Published online: 23 March 2020

\section{References}

1. Stanley D, Denman SE, Hughes RJ, Geier MS, Crowley TM, Chen H, Haring VR, Moore RJ. Intestinal microbiota associated with differential feed conversion efficiency in chickens. Appl Microbiol Biotechnol. 2012; 96:1361-9.

2. Stappenbeck TS, Hooper LV, Gordon J. Developmental regulation of intestinal angiogenesis by indigenous microbes via Paneth cells. Proc Natl Acad Sci U S A. 2002;99:15451-5

3. Talham GL, Jiang H-Q, Bos NA, Cebra JJ. Segmented filamentous bacteria are potent stimuli of a physiologically normal state of the murine gut mucosal immune system. Infect Immun. 1999;67:1992-2000.

4. Oakley BB, Lillehoj HS, Kogut MH, Kim WK, Maurer JJ, Pedroso A, et al. The chicken gastrointestinal microbiome. FEMS Microbiol Lett. 2014;360: 100-12.

5. Cui Y, Wang Q, Liu S, Sun R, Zhou Y, Li Y. Age-related variations in intestinal microflora of free-range and caged hens. Front Microbiol. 2017:8:1310.

6. Ford L, Moffatt C, Fearnley E, Miller M, Gregory J, Sloan-Gardner T, et al. The epidemiology of Salmonella enterica outbreaks in Australia, 20012016. Front Sustain Food Syst. 2018;2:86.

7. Dieye $Y$, Ameiss $K$, Mellata M, Curtiss R. The Salmonella Pathogenicity Island (SPI) 1 contributes more than SPI2 to the colonization of the chicken by Salmonella enterica serovar Typhimurium. BMC Microbiol. 2009;9:3.

8. Stecher B, Barthel M, Schlumberger MC, Haberli L, Rabsch W, Kremer M, et al. Motility allows S. Typhimurium to benefit from the mucosal defence. Cell Microbiol. 2008:10:1166-80.

9. Staib L, Fuchs TM. Regulation of fucose and 1, 2-propanediol utilization by Salmonella enterica serovar Typhimurium. Front Microbiol. 2015;6: 1116

10. Ballou AL, Ali RA, Mendoza MA, Ellis JC, Hassan HM, Croom WJ, et al. Development of the chick microbiome: how early exposure influences future microbial diversity. Front Vet Sci. 2016;3:2.

11. Mon KKZ, Saelao P, Halstead MM, Chanthavixay G, Chang H-C, Garas L, et al. Salmonella enterica serovars Enteritidis infection alters the indigenous microbiota diversity in young layer chicks. Front Vet Sci. 2015;2:61.

12. Myers SP. The causes of intestinal dysbiosis: a review. Altern Med Rev. 2004;9:180-97.

13. Morrison DJ, Preston T. Formation of short chain fatty acids by the gut microbiota and their impact on human metabolism. Gut Microbes. 2016:7:189-200

14. Sun M, Wu W, Liu Z, Cong Y. Microbiota metabolite short chain fatty acids, GPCR, and inflammatory bowel diseases. J Gastroenterol. 2017;52:1-8.

15. Fellows R, Denizot J, Stellato C, Cuomo A, Jain P, Stoyanova E, et al. Microbiota derived short chain fatty acids promote histone crotonylation in the colon through histone deacetylases. Nat Commun. 2018;9:105.

16. Scheppach W. Effects of short chain fatty acids on gut morphology and function. Gut. 1994;35:S35-8.

17. Senga T, Iwamoto S, Yoshida T, Yokota T, Adachi K, Azuma E, et al. LSSIG is a novel murine leukocyte-specific GPCR that is induced by the activation of STAT3. Blood. 2003;101:1185-7.

18. Lu Y, Fan C, Li P, Lu Y, Chang X, Qi K. Short chain fatty acids prevent highfat-diet-induced obesity in mice by regulating $G$ protein-coupled receptors and gut microbiota. Sci Rep. 2016;6:37589.

19. Zhang J-M, Sun Y-S, Zhao L-Q, Chen T-T, Fan M-N, Jiao H-C, Zhao J-P, Wang $X-J$, Li F-C, Li H-F, et al. SCFAs-induced GLP-1 secretion links the regulation of gut microbiome on hepatic Lipogenesis in chickens. Front Microbiol. 2019;10:2176.

20. Fuller R. Probiotics in man and animals. J Appl Bacteriol. 1989;66:365-

21. Lloyd AB, Cumming RB, Kent RD. Prevention of Salmonella Typhimurium infection in poultry by pretreatment of chickens and poults with intestinal extracts. Aust Vet J. 1977;53:82-7. 
22. Laport MS, Bauwens M, Collard M, George I. Phylogeny and antagonistic activities of culturable Bacteria associated with the gut microbiota of the sea urchin (Paracentrotus lividus). Curr Microbiol. 2018;75:359-67.

23. Reid G, Howard J, Gan BS. Can bacterial interference prevent infection? Trends Microbiol. 2001;9:424-8.

24. Honda K, Littman DR. The microbiome in infectious disease and inflammation. Annu Rev Immunol. 2012;30:759-95.

25. Hooper LV, Wong MH, Thelin A, Hansson L, Falk PG, Gordon Jl. Molecular analysis of commensal host-microbial relationships in the intestine. Science. 2001;291:881-4.

26. Buffie CG, Pamer EG. Microbiota-mediated colonization resistance against intestinal pathogens. Nat Rev Immunol. 2013;13:790.

27. Rechkemmer G, Rönnau K. Fermentation of polysaccharides and absorption of short chain fatty acids in the mammalian hindgut. Comp Biochem Physiol A. 1988;90:563-8.

28. Million M, Alou MT, Khelaifia S, Bachar D, Lagier JC, Dione N, et al. Increased gut redox and depletion of anaerobic and methanogenic prokaryotes in severe acute malnutrition. Sci Rep. 2016;6:26051.

29. Ismail AS, Valastyan JS, Bassler BL. A host-produced autoinducer-2 mimic activates bacterial quorum sensing. Cell Host Microbe. 2016;19:470-80.

30. Wilson $\mathrm{KH}$, Perini F. Role of competition for nutrients in suppression of Clostridium difficile by the colonic microflora. Infect Immun. 1988;56:2610-4.

31. Tejada-Simon MV, Pestka JJ. Proinflammatory cytokine and nitric oxide induction in murine macrophages by cell wall and cytoplasmic extracts of lactic acid bacteria. J Food Prot. 1999;62:1435-44.

32. Brisbin JT, Gong J, Parvizi P, Sharif S. Effects of lactobacilli on cytokine expression by chicken spleen and cecal tonsil cells. Clin Vaccine Immunol. 2010;17:1337-43.

33. Mikulski D, Jankowski J, Naczmanski J, Mikulska M, Demey V. Effects of dietary probiotic (Pediococcus acidilactici) supplementation on performance, nutrient digestibility, egg traits, egg yolk cholesterol, and fatty acid profile in laying hens. Poult Sci. 2012;91:2691-700.

34. Panda AK, Rama Rao SS, Raju MVLN, Sharma SS. Effect of probiotic (Lactobacillus sporogenes) feeding on egg production and quality, yolk cholesterol and humoral immune response of white Leghorn layer breeders. J Sci Food Agric. 2008:88:43-7.

35. Van Coillie E, Goris J, Cleenwerck I, Grijspeerdt K, Botteldoorn N, Van Immerseel $F$, et al. Identification of lactobacilli isolated from the cloaca and vagina of laying hens and characterization for potential use as probiotics to control Salmonella Enteritidis. J Appl Microbiol. 2007;102:1095-106.

36. Luoma A, Markazi A, Shanmugasundaram R, Murugesan G, Mohnl M, Selvaraj R. Effect of synbiotic supplementation on layer production and cecal Salmonella load during a Salmonella challenge. Poult Sci. 2017;96: 4208-16.

37. Wang L, Li L, Lv Y, Chen Q, Feng J, Zhao X. Lactobacillus plantarum restores intestinal permeability disrupted by Salmonella infection in newly-hatched chicks. Sci Rep. 2018:8:2229.

38. Pascual M, Hugas M, Badiola Jl, Monfort JM, Garriga M. Lactobacillus salivarius CTC2197 prevents Salmonella Enteritidis colonization in chickens. Appl Environ Microbiol. 1999;65:4981-6.

39. Pavic A, Groves PJ, Bailey G, Cox JM. A validated miniaturized MPN method, based on ISO 6579: 2002, for the enumeration of Salmonella from poultry matrices. J Appl Microbiol. 2010;109:25-34

40. Gole VC, Woodhouse R, Caraguel C, Moyle T, Rault J-L, Sexton M, et al. Dynamics of Salmonella shedding and welfare of hens in free-range egg production systems. Appl Environ Microbiol. 2017;83:e03313-6.

41. Pande W, Devon RL, Sharma P, McWhorter AR, Chousalkar KK. Study of Salmonella Typhimurium infection in laying hens. Front Microbiol. 2016;7: 203.

42. Gole VC, Chousalkar KK, Roberts JR. Survey of Enterobacteriaceae contamination of table eggs collected from layer flocks in Australia. Int J Food Microbiol. 2013;164:161-5.

43. Schloss PD, Westcott SL, Ryabin T, Hall JR, Hartmann M, Hollister EB, et al. Introducing mothur: open-source, platform-independent, community-supported software for describing and comparing microbial communities. Appl Environ Microbiol. 2009;75:7537-41.

44. MiSeq SOP [https://www.mothur.org/wiki/MiSeq_SOP]. Accessed 10 Aug 2019

45. Quast C, Pruesse E, Yilmaz P, Gerken J, Schweer T, Yarza P, et al. The SILVA ribosomal RNA gene database project: improved data processing and web-based tools. Nucleic Acids Res. 2012;41:D590-6.
46. Westcott SL, Schloss PD. OptiClust, an improved method for assigning amplicon-based sequence data to operational taxonomic units. MSphere. 2017;2:e00073-17.

47. Ondov BD, Bergman NH, Phillippy AM. Interactive metagenomic visualization in a web browser. BMC Bioinformatics. 2011;12:385.

48. Script to convert mothur taxonomy summary files to Krona XML format [https://github.com/accaldwell/mothur_krona]. Accessed 10 Aug 2019.

49. Simple script to generate Krona charts for all samples in an OTU table file [https://github.com/GenomicaMicrob/OTUsamples2krona]. Accessed 10 Aug 2019.

50. Zakrzewski M, Proietti C, Ellis JJ, Hasan S, Brion MJ, Berger B, et al. Calypso: a user-friendly web-server for mining and visualizing microbiome-environment interactions. Bioinformatics. 2016;33:782-3.

51. Chen L, Reeve J, Zhang L, Huang S, Wang X, Chen J. GMPR: A robust normalization method for zero-inflated count data with application to microbiome sequencing data. PeerJ. 2018;6:406264.

52. Pickard JM, Zeng MY, Caruso R, Núñez G. Gut microbiota: role in pathogen colonization, immune responses, and inflammatory disease. Immunol Rev. 2017;279:70-89.

53. Videnska P, Sedlar K, Lukac M, Faldynova M, Gerzova L, Cejkova D, et al Succession and replacement of bacterial populations in the caecum of egg laying hens over their whole life. PLoS One. 2014;9:e115142.

54. Azcarate-Peril MA, Butz N, Cadenas MB, Koci M, Ballou A, Mendoza M, et al. An attenuated Salmonella enterica serovar Typhimurium strain and galacto-oligosaccharides accelerate clearance of Salmonella infections in poultry through modifications to the gut microbiome. Appl Environ Microbiol. 2018;84:e02526-17

55. Morotomi M, Nagai F, Watanabe Y. Description of Christensenella minuta gen. nov., sp. nov., isolated from human faeces, which forms a distinct branch in the order Clostridiales, and proposal of Christensenellaceae fam. nov. Int J Syst Evol Microbiol. 2012;62:144-9.

56. Dey N, Soergel DA, Repo S, Brenner SE. Association of gut microbiota with post-operative clinical course in Crohn's disease. BMC Gastroenterol. 2013;13:131.

57. Liu L, Lin L, Zheng L, Tang H, Fan $X$, Xue N, et al. Cecal microbiome profile altered by Salmonella enterica, serovar Enteritidis inoculation in chicken. Gut Pathog. 2018:10:34

58. Qin J, Li R, Raes J, Arumugam M, Burgdorf KS, Manichanh C, et al. A human gut microbial gene catalogue established by metagenomic sequencing. Nature. 2010;464:59

59. Amir I, Bouvet P, Legeay C, Gophna U, Weinberger A. Eisenbergiella tayi gen. nov., sp. nov., isolated from human blood. Int J Syst Evol Microbiol. 2014;64: 907-14.

60. Mancabelli L, Milani C, Lugli GA, Turroni F, Cocconi D, van Sinderen D, et al. Identification of universal gut microbial biomarkers of common human intestinal diseases by meta-analysis. FEMS Microbiol Ecol. 2017:93:fix153.

61. Sergeant M, Constantinidou C, Cogan TA, Bedford MR, Penn CW, Pallen MJ. Extensive microbial and functional diversity within the chicken cecal microbiome. PLoS One. 2014;9:e91941.

62. Wang K, Liao M, Zhou N, Bao L, Ma K, Zheng Z, et al. Parabacteroides distasonis alleviates obesity and metabolic dysfunctions via production of succinate and secondary bile acids. Cell Rep. 2019;26:222-35 e225.

63. Polansky O, Sekelova Z, Faldynova M, Sebkova A, Sisak F, Rychlik I. Important metabolic pathways and biological processes expressed by chicken cecal microbiota. Appl Environ Microbiol. 2016:82:1569-76.

64. Berger FK, Schwab N, Glanemann M, Bohle RM, Gärtner B, Groesdonk HV. Flavonifractor (Eubacterium) plautii bloodstream infection following acute cholecystitis. IDCases. 2018;14:e00461.

65. LeBlanc JG, Chain F, Martín R, Bermúdez-Humarán LG, Courau S, Langella P. Beneficial effects on host energy metabolism of short-chain fatty acids and vitamins produced by commensal and probiotic bacteria. Microb Cell Factories. 2017:16:79. 